\title{
PROPRIEDADES REOLÓGICAS E EFEITO DA ADIÇÃO DE SAL NA VISCOSIDADE DE EXOPOLISSACARÍDEOS PRODUZIDOS POR BACTÉRIAS DO GÊNERO Rhizobium
}

\section{Gabriel Aranda-Selverio e Ana Lúcia Barretto Penna}

Departamento de Engenharia e Tecnologia de Alimentos, Instituto de Biociências, Letras e Ciências Exatas, Universidade Estadual Paulista, 15054-000 São José do Rio Preto - SP, Brasil

Luciana Frizarin Campos-Sás, Osvaldo dos Santos Junior, Ana Flora Dalberto Vasconcelos e Maria de Lourdes Corradi da Silva* Departamento de Física, Química e Biologia, Faculdade de Ciências e Tecnologia, Universidade Estadual Paulista, CP 266, 19060-900 Presidente Prudente - SP, Brasil

\section{Eliana Gertrudes Macedo Lemos e João Carlos Campanharo}

Departamento de Tecnologia, Faculdade de Ciências Agrárias e Veterinárias, Universidade Estadual Paulista, 14884-900 Jaboticabal - SP, Brasil

Joana Léa Meira Silveira

Departamento de Bioquímica e Biologia Molecular, Universidade Federal do Paraná, CP 19046, 81531990 Curitiba - PR, Brasil

Recebido em 21/7/09; aceito em 8/12/09; publicado na web em 26/3/10

\begin{abstract}
RHEOLOGICAL PROPERTIES AND EFFECT OF THE SALT ADDITION ON THE EXOPOLYSACCHARIDES VISCOSITY PRODUCED BY BACTERIA OF Rhizobium GENUS. Viscosity of some polysaccharide solutions supports that these molecules can be applied in food sectors. Four exopolysaccharides $\left(\mathrm{R}_{1}, \mathrm{R}_{2}, \mathrm{R}_{3}, \mathrm{R}_{4}\right)$ produced by different Rhizobium strains were selected. Sugar composition and differences in the uronic acid contents suggests that chemical structure of these molecules can vary when different culture conditions and strains are analyzed. The Power Law was the rheological model used to represent the experimental data of shear stress versus shear rate. All exopolysaccharides showed non-Newtonian behavior, with pseudoplastic characteristics. $R_{1}, R_{2}$ and $\mathrm{R}_{4}$ showed a slight increase in viscosity in the presence of $0,2 \mathrm{M} \mathrm{NaCl}$.
\end{abstract}

Keywords: rheological behaviour; exopolysaccharides from Rhizobium; pseudoplastic solution.

\section{INTRODUÇÃO}

Há na natureza plantas e micro-organismos que secretam polímeros, denominados exopolissacarídeos (EPS), que podem ser classificados como homo- e heteropolissacarídeos. Os primeiros são, geralmente, glucanas neutras, enquanto os heteropolissacarídeos são, em sua grande maioria, polianiônicos devido à presença de ácido urônico.

Os EPS microbianos são considerados substitutos ideais para polissacarídeos de plantas, pois uma vez estabelecidas as melhores condições de cultivo as moléculas produzidas apresentam características químicas e físicas constantes e, geralmente, superiores às gomas naturais. ${ }^{1}$ A aplicação desses biopolímeros em diferentes setores industriais, tais como alimentício e farmacêutico, ${ }^{2}$ deve-se ao fato de alterarem as propriedades físicas do meio onde se encontram, seja por conferirem alta viscosidade às soluções ou por criarem redes intermoleculares coesivas. Na indústria de alimentos podem ser utilizados como espessantes, estabilizantes, emulsificantes, coagulantes, formadores de filmes, gelificantes, agentes de suspensão e dispersantes. ${ }^{3}$

As características físico-químicas dos polissacarídeos dependem da sequência em que os açúcares estão dispostos na molécula, da presença de resíduos iônicos e da forma como as cadeias se entrelaçam. ${ }^{2}$ A solubilidade dos polímeros tende a ser maior quando grupos ácidos ionizáveis estão presentes na molécula. Soluções aquosas de polissacarídeos contendo sais inorgânicos podem ter suas viscosidades modificadas e, por esta razão, os estudos reológicos normalmente investigam o comportamento dos biopolímeros em tais condições. ${ }^{4}$

Polissacarídeos microbianos, tais como xantana, gelana, alginatos, celulose bacteriana, ácido hialurônico e succinoglucanos, já são aplicados nas indústrias de alimentos, química e farmacêutica. ${ }^{1,5}$

*e-mail: corradi@fct.unesp.br
A aplicação da goma xantana na indústria de alimentos deve-se principalmente ao seu comportamento não-Newtoniano e formação de soluções pseudoplásticas a baixas concentrações; ${ }^{6}$ essas características reológicas são dependentes da concentração do polímero e da presença de eletrólitos em solução. ${ }^{7}$

Dentre os micro-organismos produtores de polissacarídeos há um gênero de bactérias, denominado genericamente de rizóbio, que secreta exopolissacarídeos com diferentes funções biológicas. ${ }^{8}$ Essas moléculas são necessárias no processo de invasão da célula hospedeira e na formação de nódulos fixadores de nitrogênio em plantas leguminosas. Um exemplo típico dessa interação envolve a bactéria gram-negativa, do solo, Sinorhizobium meliloti e seu hospedeiro, alfafa. A bactéria secreta vários polissacarídeos entre eles succinoglucanas e galactoglucanas, necessários para a invasão da mesma. ${ }^{9}$

Castellane e Lemos ${ }^{10}$ cultivaram Rhizobium tropici SEMIA 4077 e SEMIA 4080, em diferentes fontes de carbono, para produção de exopolissacarídeos e observaram que eram constituídos, majoritariamente, por resíduos de glicose e galactose, com traços de manose, ramnose e ácidos urônicos. Entretanto, as proporções de açúcar se mostraram variáveis para cada exopolissacarídeo produzido. Considerando-se que as propriedades físico-químicas podem variar em função da composição química da molécula, este trabalho teve como objetivo investigar o comportamento reológico dos exopolissacarídeos produzidos pelas diferentes linhagens do Rhizobium.

\section{PARTE EXPERIMENTAL}

\section{Obtenção dos exopolissacarídeos e preparo de suas soluções}

Os exopolissacarídeos utilizados para as análises foram fornecidos pela Profa. Dra. E. G. de M. Lemos, Departamento de Tecnologia, 
Faculdade de Ciências Agrárias e Veterinárias, UNESP, campus de Jaboticabal. Detalhes das condições de cultivo dos micro-organismos produtores destes EPS não foram disponibilizados. Estas informações estão sob sigilo de patente com registro PI0304053-4.

Três diferentes linhagens de bactérias do gênero Rhizobium foram utilizadas para a produção dos exopolissacarídeos. Os EPS $\mathrm{R}_{1}$ e $\mathrm{R}_{2}$ foram produzidos a partir da mesma estirpe, Rhizobium tropici, com diferenças nas condições de cultivo (dados não disponíveis). O EPS $\mathrm{R}_{3}$ foi produzido a partir de uma linhagem denominada Mesorhizobium e o EPS $\mathrm{R}_{4}$ a partir de uma estirpe selecionada conhecida como Rhizobium sp.

Antes de todas as análises os exopolissacarídeos foram, individualmente, dialisados (72 h em água destilada corrente) em tubos de $12 \mathrm{KDa}$, concentrados a baixa temperatura $\left(38^{\circ} \mathrm{C}\right)$ e liofilizados (Figura 1S, material suplementar). Foram mantidos desta forma até o momento do uso.

\section{Métodos analíticos}

Açúcares totais foram determinados pelo método do fenol-ácido sulfúrico ${ }^{11}$ e os açúcares redutores pelo método de Somogy e Nelson. ${ }^{12}$ A glucose foi utilizada como padrão em ambos os ensaios. Os ácidos urônicos foram quantificados pelo método de Filisetti-Cozzi e Carpita ${ }^{13} \mathrm{e}$ o ácido glucurônico foi utilizado como padrão. As proteínas foram determinadas pelo método de Bradford ${ }^{14} \mathrm{e}$ soro albumina bovina foi utilizado como padrão.

\section{Hidrólise ácida total}

Para se determinar a composição em monossacarídeos, cada EPS (50 $\mu \mathrm{g}$ em açúcares totais) foi submetido à hidrólise ácida ${ }^{15}$ (TFA 5 $\mathrm{M}, 100{ }^{\circ} \mathrm{C}, 12 \mathrm{~h}$ ), em tubo selado. Após hidrólise, o excesso de ácido foi removido por codestilação com água e analisado por HPAEC-PAD (high performance anionic exchange chromatogaraphy-pulsed amperometric detection). As condições da análise cromatográfica foram efetuadas como descrito por Mendes e colaboradores. ${ }^{16}$

\section{Comportamento reológico}

Para estudo do comportamento reológico os EPS foram solubilizados em água deionizada à temperatura de $\pm 20^{\circ} \mathrm{C}$, nas concentrações de 2,5 e $10 \mathrm{~g} / \mathrm{L}$. As soluções foram mantidas sob agitação mecânica constante, em velocidade média, à temperatura de $\pm 20^{\circ} \mathrm{C}$ por $18 \mathrm{~h}$.

As medidas reológicas das soluções polissacarídicas foram efetuadas em reômetro Haake Rheostress, modelo RS75, acoplado a um banho termostatizado Haake K15 e termocirculador de água DC5B3. Foi utilizado o sensor cone-placa com diâmetro de $60 \mathrm{~mm}$ e ângulo do cone de $2^{\circ}$. A taxa de cisalhamento ou deformação $(\dot{\gamma})$ variou de 0 até $200 \mathrm{~s}^{-1}$ com um tempo de $300 \mathrm{~s}$, com aquisição de 200 pontos em escala logarítmica. As amostragens foram realizadas em triplicata à temperatura de $25^{\circ} \mathrm{C}$. Os reogramas, para cada uma das três concentrações de cada EPS, foram gerados utilizando-se software RheoWin (versão 3.20). O modelo da Lei da Potência (Equação 1) foi ajustado aos reogramas e os valores do índice de consistência e índice de comportamento de fluxo do fluido foram determinados para as concentrações das soluções analisadas

$\tau=\mathrm{K} \cdot \dot{\gamma}^{\mathrm{n}}$

onde: $\tau=$ tensão de cisalhamento $(\mathrm{Pa}) ; \dot{\gamma}=$ taxa de deformação $\left(\mathrm{s}^{-1}\right)$; $\mathrm{K}=$ índice de consistência (Pa.s); $\mathrm{n}=$ índice de comportamento de fluxo do fluido (adimensional)

\section{Influência da salinidade na viscosidade}

Com o objetivo de avaliar o comportamento reológico dos biopolímeros na presença de sais, os mesmos foram solubilizados em $\mathrm{NaCl}$ $0,2 \mathrm{M}$ na concentração de $5 \mathrm{~g} / \mathrm{L}$ à temperatura de $25^{\circ} \mathrm{C}$ e as medidas reológicas foram efetuadas como no item Comportamento reológico.

\section{RESULTADOS E DISCUSSÃO}

\section{Quantificação dos exopolissacarídeos}

Os exopolissacarídeos, $\mathrm{R}_{1}, \mathrm{R}_{2}, \mathrm{R}_{3}$ e $\mathrm{R}_{4}$, foram quantificados para açúcares totais e redutores, proteínas e ácidos urônicos. Os resultados são mostrados na Tabela 1. Os baixos valores encontrados na quantificação de açúcares redutores ${ }^{12}$ sinalizam que os carboidratos presentes em solução estão, principalmente, na forma de polissacarídeos e o elevado teor de ácido urônico $(8,4 \%)$ presente no polissacarídeo produzido pelo Mesorhizobium $\left(\mathrm{R}_{3}\right)$ sugere a presença de um polímero aniônico. No preparo das soluções polissacarídicas para as reações de quantificação o $R_{3}$ apresentou-se como menos viscoso e, de acordo com a literatura, o comportamento reológico de polímeros acídicos é diferente daquele apresentado pelos polímeros neutros. A presença de ácidos glucurônico e pirúvico na goma xantana confere ionicidade à mesma, promovendo alterações na conformação molecular e aumentando a solubilidade de suas soluções. ${ }^{4}$

Tabela 1. Quantificações de açúcares totais e redutores, proteínas e ácido urônico dos exopolissacarídeos produzidos por diferentes linhagens de bactérias do gênero Rhizobium

\begin{tabular}{|c|c|c|c|c|c|c|}
\hline \multirow{2}{*}{ 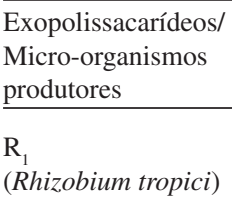 } & \multirow{2}{*}{$\begin{array}{c}\begin{array}{c}\text { Açúcar } \\
\text { total }(\%)\end{array} \\
94,6\end{array}$} & \multirow{2}{*}{$\begin{array}{c}\begin{array}{c}\text { Açúcar } \\
\text { redutor } \\
(\%)\end{array} \\
0,10\end{array}$} & \multirow{2}{*}{$\begin{array}{c}\text { Proteína } \\
(\%)\end{array}$} & \multirow{2}{*}{$\begin{array}{c}\text { Ácido } \\
\text { urônico } \\
(\%) \\
2,4\end{array}$} & \multicolumn{2}{|c|}{$\begin{array}{l}\text { Composição } \\
\text { em açúcares } \\
\text { neutros (\%) }\end{array}$} \\
\hline & & & & & $\begin{array}{l}\text { Glc } \\
\text { Gal } \\
\text { Man }\end{array}$ & $\begin{array}{r}77 \\
18 \\
3\end{array}$ \\
\hline $\begin{array}{l}\mathrm{R}_{2} \\
\text { (Rhizobium tropici) }\end{array}$ & 94,1 & 0,11 & 4,2 & 1,7 & $\begin{array}{r}\text { Glc } \\
\text { Gal } \\
\text { Man }\end{array}$ & $\begin{array}{c}79 \\
21 \\
\text { traços }\end{array}$ \\
\hline $\begin{array}{l}\mathrm{R}_{3} \\
\text { (Mesorhizobium) }\end{array}$ & 87,1 & 0,12 & 4,5 & 8,4 & $\begin{array}{r}\text { Glc } \\
\text { Gal } \\
\text { Man }\end{array}$ & $\begin{array}{c}87 \\
13 \\
\text { traços }\end{array}$ \\
\hline $\begin{array}{l}\mathrm{R}_{4} \\
\text { (Rhizobium sp.) }\end{array}$ & 95,1 & 0,10 & 4,1 & 0,8 & $\begin{array}{l}\text { Glc } \\
\text { Gal } \\
\text { Man }\end{array}$ & $\begin{array}{r}80 \\
20 \\
\operatorname{tracos}\end{array}$ \\
\hline
\end{tabular}

Traços significam $<1 \%$

Os resultados da hidrólise ácida, para açúcares neutros (Tabela 1), indicaram uma pequena diferença na composição de $\mathrm{R}_{1}$ e $\mathrm{R}_{2}$, ambos produzidos pelo mesmo micro-organismo, Rhizobium tropici, com pequenas variações no método de produção. A presença majoritária da glucose em todos os exopolissacarídeos indica uma cadeia principal constituída, principalmente, de glucose. De acordo com a literatura, micro-organismos de linhagens diferentes ou com diferenças apenas nas condições de cultivo produzem polissacarídeos semelhantes nos quais a cadeia principal, normalmente, é a mesma, com variações no grau de ramificação. Esses resultados também foram, parcialmente, demonstrados por Castellane e Lemos. ${ }^{10}$

Considerando-se que os resultados apresentados na Tabela 1 indicam que as moléculas devem possuir algumas diferenças estruturais, foram preparadas soluções de diferentes concentrações para 
averiguar o comportamento reológico de cada exopolissacarídeo, selecionado para esse trabalho.

\section{Comportamento reológico das soluções formadas pelos exopolissacarídeos}

A curva de fluxo, definida a partir das medidas de tensão de cisalhamento $(\tau)$ x e taxa de deformação $(\dot{\gamma})$, permite verificar o comportamento de fluxo das soluções polissacarídicas. A partir da construção do reograma é possível estabelecer se um líquido apresenta comportamento de fluxo Newtoniano ou não-Newtoniano, em determinadas condições de tensão ou taxa de deformação. Um líquido Newtoniano é representado, graficamente, por uma reta com início na origem da curva de fluxo. Os que não exibem esse comportamento são chamados não-Newtonianos. A análise dos reogramas com diferentes concentrações poliméricas permite evidenciar a transição de um comportamento Newtoniano para não-Newtoniano. ${ }^{17}$

$\mathrm{O}$ perfil apresentado nos reogramas mostrados na Figura 1 permite estabelecer que as soluções polissacarídicas $R_{1} R_{2} R_{3}$ e $R_{4}$ têm comportamento de fluxo de líquidos não-Newtonianos. A diminuição no crescimento da tensão com o aumento da taxa de cisalhamento, resultando em menor viscosidade aparente, caracteriza as soluções como fluidos pseudoplásticos. ${ }^{18}$ Soluções formadas pelo EPS 450, produzido pelo Bacillus sp, também apresentam comportamento pseudoplástico assim como várias outras soluções de polissacarídeos microbianos. ${ }^{19}$

A descrição do comportamento reológico dos materiais pode ser feita através de modelos que relacionam como a tensão de cisalhamento varia com a taxa de deformação. Dentre os modelos matemáticos existentes, alguns dos mais utilizados para os sistemas de alimentos são OstwaldDe-Waele (Lei da Potência), Casson, Herschel-Bulkley e Mizrahi-Berki. Os dois primeiros utilizam equações matemáticas com dois parâmetros e os demais com três parâmetros. ${ }^{20}$ Para as soluções formadas pelos exopolissacarídeos produzidos pelas diferentes linhagens de Rhizobium, nas concentrações de 2,5 e $10 \mathrm{~g} / \mathrm{L}$ o modelo de Ostwald-De-Waele proporcionou os melhores ajustes, com maiores valores do coeficiente de regressão $\left(\mathrm{R}^{2}\right)$ e menores valores para $\chi^{2}$ (Tabela 2$)$.

Tabela 2. Parâmetros reológicos obtidos pela análise das soluções exopolissacarídicas pela aplicação do modelo da Lei da Potência

\begin{tabular}{lccccc}
\hline Amostra & $\begin{array}{c}\text { Concentração } \\
(\mathrm{g} / \mathrm{L})\end{array}$ & $\begin{array}{c}\text { Índice de } \\
\text { consistência } \\
(\mathrm{K})\end{array}$ & $\begin{array}{c}\text { Índice de } \\
\text { de fluxo }(\mathrm{n})\end{array}$ & $\begin{array}{c}\text { Coeficiente } \\
\text { de regressão } \\
\left(\mathrm{R}^{2}\right)\end{array}$ & $\chi^{2}$ \\
\hline EPS R $_{1}$ & 2 & 0.51 & 0.30 & 0,99 & 0,05 \\
& 5 & 3.14 & 0.11 & 0,98 & 0,08 \\
& $5 \mathrm{em} \mathrm{NaCl}$ & 3.36 & 0.19 & 0,99 & 0,04 \\
& 10 & 9.30 & 0.12 & 0,99 & 0,05 \\
& & & & & \\
EPS R & & 0.52 & 0.30 & 0,99 & 0,09 \\
& 5 & 3.70 & 0.09 & 0,99 & 0,06 \\
& $5 \mathrm{em} \mathrm{NaCl}$ & 3.80 & 0.17 & 0,99 & 0,08 \\
& 10 & 7.33 & 0.14 & 0,99 & 0.06 \\
EPS R $_{3}$ & 2 & 0.04 & 0.73 & 0,99 & 0,06 \\
& 5 & 0.52 & 0.50 & 0,99 & 0,06 \\
& $5 \mathrm{em} \mathrm{NaCl}$ & 0.32 & 0.55 & 0,99 & 0.06 \\
& 10 & 4.90 & 0.28 & 0,99 & 0,07 \\
& & & & & \\
EPS R $_{4}$ & 2 & 0.50 & 0.32 & 0,99 & 0,06 \\
& 5 & 3.24 & 0.10 & 0,98 & 0,09 \\
& $5 \mathrm{em} \mathrm{NaCl}$ & 4,31 & 0.21 & 0,99 & 0,02 \\
& 10 & 7.13 & 0.13 & 0,99 & 0,04 \\
\hline
\end{tabular}

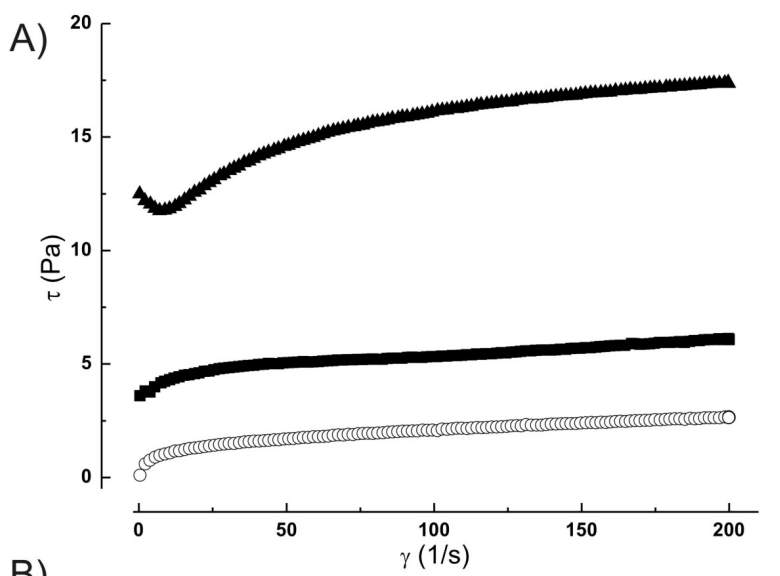

B)

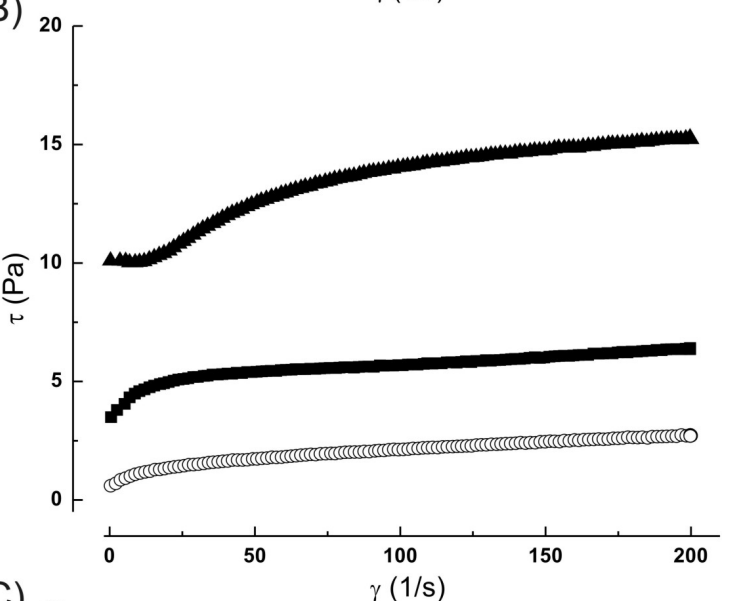

C)

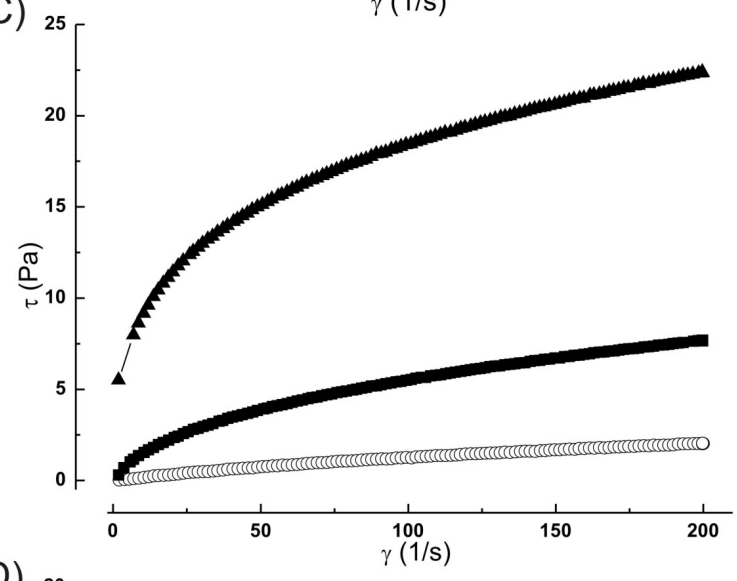

D)

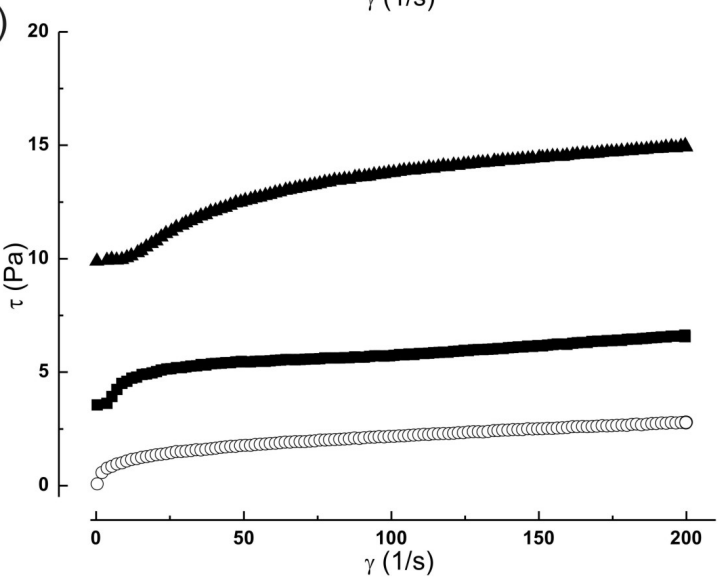

Figura 1. Reogramas (tensão de cisalhamento x taxa de deformação) das soluções polissacarídicas $(A) R_{p},(B) R_{2},(C) R_{3} e(D) R_{4}$ nas concentrações de $2 \mathrm{~g} / \mathrm{L}(-\mathrm{O}-), 5 \mathrm{~g} / \mathrm{L}(-\mathbf{-}-)$ e $10 \mathrm{~g} / \mathrm{L}\left(-\mathbf{\Delta}_{-}\right)$, à temperatura de $25^{\circ} \mathrm{C}$ 
O desvio de " $n$ " da unidade indica o grau de desvio do comportamento newtoniano. Valores de $\mathrm{n}<1$ definem comportamento pseudoplástico. As soluções polissacarídicas estudadas neste trabalho apresentaram $\mathrm{n}<1$, indicando comportamento pseudoplástico (Tabela 2).

É possível, ainda, observar a partir do índice de consistência (K) (Tabela 2) um aumento progressivo da viscosidade conforme se aumenta a concentração das soluções de todos EPS. Nota-se uma tendência da solução do exopolissacarídeo $\mathrm{R}_{1}$ ser mais viscosa do que as soluções de $R_{2}, R_{3}$ e $R_{4}$, principalmente em concentrações maiores $(10 \mathrm{~g} / \mathrm{L}) . \mathrm{R}_{3}$ apresenta menores valores de $\mathrm{K}$, em qualquer uma das concentrações analisadas, em comparação aos demais EPS.

A Tabela 2 mostra que as soluções dos exopolissacarídeos $R_{1}$, $\mathrm{R}_{2}$ e $\mathrm{R}_{4}$, em presença de $\mathrm{NaCl} 0,2 \mathrm{M}$, apresentam um pequeno aumento no valor do índice de consistência (K) quando comparados com as mesmas concentrações das soluções aquosas dos respectivos polissacarídeos, indicando que a presença desse eletrólito favorece a viscosidade dos polímeros. Um valor menor de $\mathrm{K}$ para o exopolissacarídeo produzido pelo Mesorhizobium $\left(\mathrm{R}_{3}\right)$, em presença de sal na concentração de $5 \mathrm{~g} / \mathrm{L}$ pode ser atribuído à interação entre resíduos carregados de ácido urônico $(8,4 \%)$ e os íons provenientes da dissociação do sal. A dissolução do hidrogel quitosana-xantana em meio eletrolítico promove o aumento da viscosidade, enquanto para a goma xantana sozinha, em baixas concentrações, a adição de um eletrólito, por exemplo, $\mathrm{NaCl}$, reduz a viscosidade; entretanto, em concentrações elevadas a presença do eletrólito tem efeito oposto, embora um gel fraco de xantana seja formado. ${ }^{7,21}$

\section{Efeito da adição de sal na viscosidade}

As curvas de fluxo têm suas correspondentes curvas de viscosidade. ${ }^{17}$ Foram realizados estudos quanto à viscosidade aparente das soluções aquosas $(2,5$ e $10 \mathrm{~g} / \mathrm{L})$ dos biopolímeros $\mathrm{R}_{1}, \mathrm{R}_{2}, \mathrm{R}_{3} \mathrm{e}$ $\mathrm{R}_{4}$. As medidas da viscosidade $(\eta) \mathrm{x}$ taxa de deformação $\left(\gamma^{\bullet}\right)$ foram reunidas em um único gráfico, para cada um dos EPS investigados. Pelos resultados obtidos (Figuras $2 \mathrm{~S}$ e $3 \mathrm{~S}$, material suplementar) é possível observar que a viscosidade aumenta com o aumento da concentração dos polímeros, entretanto com o aumento da taxa de deformação, para uma mesma concentração do polímero, ocorre a diminuição da viscosidade. Fluidos que sofrem diminuição da viscosidade, quando a taxa de deformação aumenta, são chamados pseudoplásticos. ${ }^{22}$ Esse perfil caracteriza o comportamento pseudoplástico de $\mathrm{R}_{1}, \mathrm{R}_{2}, \mathrm{R}_{3}$ e $\mathrm{R}_{4}$.

Um estudo comparativo da viscosidade das soluções aquosa e salina dos biopolímeros (5 g/L), juntamente com o teor de ácidos urônicos, é apresentado na Tabela 3. Até taxas de deformação de aproximadamente $2 \mathrm{~s}^{-1}$ não houve mudanças significativas nos valores de viscosidade para os quatro EPS em solução salina. Esse comportamento pode ter ocorrido devido às baixas taxas de deformação não serem suficientes para superar o movimento browniano das moléculas e orientá-las na direção do fluxo. Nessas condições, a viscosidade independe da taxa de deformação. ${ }^{17}$ Valores maiores de viscosidade são encontrados em baixas taxas de deformação $\left(10 \mathrm{~s}^{-1}\right)$, com $\mathrm{R}_{3}$ apresentando os menores valores. O decréscimo da viscosidade aparente com o aumento da taxa de deformação pode ser atribuído ao surgimento de deformações nas moléculas de polímero, produzindo formas mais alongadas, como consequência das forças hidrodinâmicas geradas no cisalhamento e/ou a um maior alinhamento das moléculas de polímero ao longo das linhas de fluxo, na direção da tensão aplicada. ${ }^{23}$ Em taxas de deformação mais elevadas, os valores de viscosidade para os quatro polímeros são praticamente semelhantes, provavelmente devido à ação mais efetiva do cisalhamento sobre a forma das moléculas, nivelando-as a uma mesma conformação limite.
Tabela 3. Viscosidade aparente ( $\eta$ ) das soluções aquosa e salina dos EPS $R_{1}$, $\mathrm{R}_{2}, \mathrm{R}_{3}$ e $\mathrm{R}_{4}$, nas concentrações de $5 \mathrm{~g} / \mathrm{L}$ e nas taxas de deformação $(\dot{\gamma}) 10 ; 50$ e $100\left(\mathrm{~s}^{-1}\right)$, à temperatura de $25^{\circ} \mathrm{C}$

\begin{tabular}{|c|c|c|c|c|c|}
\hline & \multirow{2}{*}{\multicolumn{2}{|c|}{ Ácido urônico (\%) }} & \multicolumn{3}{|c|}{ Viscosidade $(\eta)($ Pa.s) } \\
\hline & & & $10 \mathrm{~s}^{-1}$ & $50 \mathrm{~s}^{-1}$ & $100 \mathrm{~s}^{-1}$ \\
\hline \multirow[t]{2}{*}{$\mathrm{R}_{1}$} & 2,4 & $5 \mathrm{~g} / \mathrm{L} \mathrm{em} \mathrm{H} \mathrm{H}_{2} \mathrm{O}$ & 0,41 & 0,10 & 0,05 \\
\hline & & $5 \mathrm{~g} / \mathrm{L} \mathrm{em} \mathrm{NaCl} 0,2 \mathrm{M}$ & 0,51 & 0,14 & 0,08 \\
\hline \multirow[t]{2}{*}{$\mathrm{R}_{2}$} & 1,7 & $5 \mathrm{~g} / \mathrm{L} \mathrm{em} \mathrm{H}_{2} 0$ & 0,45 & 0,10 & 0,05 \\
\hline & & $5 \mathrm{~g} / \mathrm{L} \mathrm{em} \mathrm{NaCl} 0,2 \mathrm{M}$ & 0,54 & 0,14 & 0,08 \\
\hline \multirow[t]{2}{*}{$\mathrm{R}_{3}$} & 8,4 & $5 \mathrm{~g} / \mathrm{L} \mathrm{em} \mathrm{H}_{2} 0$ & 0,14 & 0,07 & 0,05 \\
\hline & & $5 \mathrm{~g} / \mathrm{L} \mathrm{em} \mathrm{NaCl} 0,2 \mathrm{M}$ & 0,10 & 0,05 & 0,04 \\
\hline \multirow[t]{2}{*}{$\mathrm{R}_{4}$} & 0,8 & $5 \mathrm{~g} / \mathrm{L} \mathrm{em} \mathrm{H} \mathrm{H}_{2} \mathrm{O}$ & 0,44 & 0,10 & 0,05 \\
\hline & & $5 \mathrm{~g} / \mathrm{L} \mathrm{em} \mathrm{NaCl} 0,2 \mathrm{M}$ & 0,44 & 0,12 & 0,07 \\
\hline
\end{tabular}

$\mathrm{R}_{1}, \mathrm{R}_{2}$ e $\mathrm{R}_{4}$ solubilizados em solução aquosa de $\mathrm{NaCl}$ apresentaram maior viscosidade quando comparados com os respectivos polímeros dissolvidos apenas em água. $\mathrm{R}_{3}$ comportou-se como o menos viscoso, tanto em meio aquoso quanto em solução aquosa contendo $\mathrm{NaCl}$, provavelmente devido ao maior percentual de ácidos urônicos $(8,4 \%)$ em sua estrutura. A adição de sais inorgânicos às soluções polissacarídicas pode promover modificações, aumentando ou diminuindo a viscosidade das soluções. ${ }^{4}$ A variação no grau dos substituintes de um polissacarídeo altera suas propriedades reológicas em solução, cujo comportamento pode ser atribuído à interação entre resíduos carregados de ácido urônico e dos íons provenientes da dissociação do sal, quando presentes em solução. ${ }^{21,24}$ Este comportamento pode indicar que uma cadeia mais distendida favorece a solubilidade e conduz a uma menor viscosidade, pois a densidade de cargas (número de cargas por unidade de comprimento) modifica as propriedades macromoleculares em solução. ${ }^{25}$ Freitas e colaboradores ${ }^{26}$ estudaram o comportamento reológico de um exopolissacarídeo acídico produzido por Pseudomonas oleovorans NRRLB-14682, em solução aquosa, e constataram que possui propriedades viscoelásticas similares à goma guar, mas com grande afinidade aos sais, como resultado de sua característica de polieletrólito.

\section{CONCLUSÕES}

Os resultados encontrados indicam que linhagens diferentes $\left(\mathrm{R}_{1} / \mathrm{R}_{2} / \mathrm{R}_{4}\right.$ e $\left.\mathrm{R}_{3}\right)$ de um mesmo micro-organismo produzem soluções polissacarídicas com propriedades reológicas (comportamento de fluxo e viscosidade) distintas, sendo as quantidades de ácido urônico, provavelmente, um dos fatores responsáveis por essa diferença.

\section{AGRADECIMENTOS}

Ao $\mathrm{CNPq}$ pela bolsa de mestrado ao aluno G. A. Selverio e à FAPESP (processo 05/53879-3).

\section{REFERÊNCIAS}

1. Singh, R. S.; Saini, G. K.; Kennedy, J. F.; Carbohydr. Polym. 2008, 73, 515.

2. Kumar, A. S.; Mody, K.; Jha, B.; J Basic Microbiol. 2007, 47, 103; Vijayendra, S. V. N.; Palavinel, G.; Mahadevamma, S.; Tharanathan, R. N.; Carbohydr. Polym. 2008, 72, 300.

3. Lopes, L.; Andrade, C. T.; Mano, E. B.; Ciênc. Hoje 1991, 12, 65; Maia, L. H.; Porte, A.; Souza, V. F.; B. CEPPA 2000, 18, 105; Araujo, L.; Alves, F.; Brasil Alimentos 2003, 19, 18; Cunha, M. A. A.; Santos, J. C.; Gómez, R. J. H. C.; Silva, S. S.; Biotecnol. Ciênc. e Desenvol. 2004, 33, 55; Gómez, M.; Ronda, F.; Caballero, P. A.; Blanco, C. A.; Rosell, C. M.; Food Hydrocoll. 2007, 21,167. 
4. Diaz, P. S.; Vendruscolo, C. T.; Vendruscolo, J. L. S.; Semin. 2004, 25 , 15 .

5. Kumar, C. G.; Joo, H-S.; Choi, J-W.; Koo, Y-M.; Chang, C-S.; Enzyme Microb. Technol. 2004, 34, 673; Piermaria, J. A.; Canal, M. L.; Abraham, A. G.; Food Hydrocoll. 2008, 22, 1520.

6. Shobha, M. S.; Tharanathan, R. N.; Food Hydrocoll. 2009, 23, 749.

7. Martínez-Ruvalcaba, A.; Chornet, E.; Rodrigues, D.; Carbohydr. Polym. 2007, 67, 586.

8. Marczak, M.; Mazur, A.; Gruszecki, W. I.; Skorupska, A.; Biochimie 2008, 90, 1781.

9. Sharypova, L. A.; Chataigné, G.; Fraysse, N.; Becker, A.; Poinsot, V.; Glycobiology 2006, 16, 1181; Serrato, R. V.; Sassaki, G. L.; Gorin, P. A. J.; Cruz, L. M.; Pedrosa, F. O.; Choudhury, B.; Carlson, R. W.; Iacomini, M.; Carbohydr. Polym. 2008, 73, 564.

10. Castellane, T. C. L.; Lemos, E. G. M. Pesqui. Agropecu. Bras. 2007, 42, 1503.

11. Dubois, N.; Gilles, K. A.; Hamilton, J. K.; Rebers, P. A., Smith, F., Anal. Chem. 1956, 28, 350.

12. Somogyi, M.; J. Biol. Chem. 1945,160, 61; Nelson, N. A.; J. Biochem. 1944, 153, 376

13. Filisetti-Cozzi, T. M. C.; Carpita, N. C.; Anal. Biochem. 1991, 197, 157.

14. Bradford, M. M.; Anal. Biochem. 1976, 72, 248.

15. Vasconcelos, A. F. D.; Monteiro, N. K.; Dekker, R. F. H.; Barbosa, A. M.; Carbonero, E. R.; Silveira, J. L. M.; Sassaki, G. L.; Silva, R.; Corradi da Silva, M. L.; Carbohydr. Res. 2008, 343, 2481.

16. Mendes, S. F.; Santos Junior, O.; Barbosa, A. M.; Vasconcelos, A. F. D.; Aranda-Selverio, G., Monteiro, N. K., Dekker, R. F. H., Pereira, M. S., Tovar, A. M. F., Mourão, P. A. S.; Corradi da Silva, M. L.; Int. J. Biol. Macrom. 2009, 45, 305.
17. Schramm, G. Em Reologia e Reometria: Fundamentos teóricos e práticos; Mothé, C.; Correia, D. Z.; Petri, H. M.; Gonçalves, M.; Carestiato, T.. eds.; Artliber Ed. Ltda: São Paulo, 2006, caps. 1, 2 e 3.

18. Navarro, R. F.; Fundamentos de reologia de polímeros, Ed. da Univ. de Caxias do Sul: Caxias do Sul, 1997.

19. Rottava, I.; Dissertação de Mestrado, Universidade Regional Integrada do Alto Araguaia e das Missões, Brasil, 2005.

20. Haminiuk, C. W. I.; Sierakowski, M. R.; Izidoro, D. R.; Masson, M. L.; Braz. J. Food Technol. 2006, 9, 291.

21. Carrington, S.; Odell, J.; Fisher, L.; Mitchell, J.; Hartley, L.; Polymer 1996, 37, 2871.

22. Bongiovani, R. A. M.; Silveira, J. L. M.; Penna, A. L. B.; Dekker, R. F. H.; Barbosa, A. M.; Corradi da Silva, M. L.; Braz. J. Food Technol. 2009, 12, 53.

23. Ferreira, G. M.; Guimarães, M. J. O. C.; Maia, M. C. A.; Rev. Bras. Frutic. 2008, 30, 385

24. Shimada, A.; Nakata, H.; Nakamura, I.; J. Ferment. Bioengineer. 1997, $84,113$.

25. Rinaudo, M. Em The relation between the chemical structure of polysaccharides and their physical properties; Philips, G. O.; Wedlack, D. J.; Willians, P. A., eds.; Gums Stabilizers Food Industries: falta cidade, 1992, p. 51-61; Milas, M. Em Polieletrólitos; De Goote, R. A. M. C.; Curvelo, A. A. S., eds.; Ed. USP: falta cidade, 1991, p. 84.

26. Freitas, F.; Alves, V. D.; Pais, J.; Costa, N.; Oliveira, C.; Mafra,L.; Hilliou, L.; Oliveira, R.; Reis, M. A. M.; Bioresour. Technol. 2009, 100, 859. 


\section{PROPRIEDADES REOLÓGICAS E EFEITO DA ADIÇÃO DE SAL NA VISCOSIDADE DE EXOPOLISSACARÍDEOS PRODUZIDOS POR BACTÉRIAS DO GÊNERO Rhizobium}

Gabriel Aranda-Selverio e Ana Lúcia Barretto Penna

Departamento de Engenharia e Tecnologia de Alimentos, Instituto de Biociências, Letras e Ciências Exatas, Universidade Estadual Paulista, 15054-000 São José do Rio Preto - SP, Brasil

Luciana Frizarin Campos-Sás, Osvaldo dos Santos Junior, Ana Flora Dalberto Vasconcelos e Maria de Lourdes Corradi da Silva* Departamento de Física, Química e Biologia, Faculdade de Ciências e Tecnologia, Universidade Estadual Paulista, CP 266, 19060-900 Presidente Prudente - SP, Brasil

\section{Eliana Gertrudes Macedo Lemos e João Carlos Campanharo}

Departamento de Tecnologia, Faculdade de Ciências Agrárias e Veterinárias, Universidade Estadual Paulista, 14884-900 Jaboticabal - SP, Brasil

Joana Léa Meira Silveira

Departamento de Bioquímica e Biologia Molecular, Universidade Federal do Paraná, CP 19046, 81531990 Curitiba - PR, Brasil

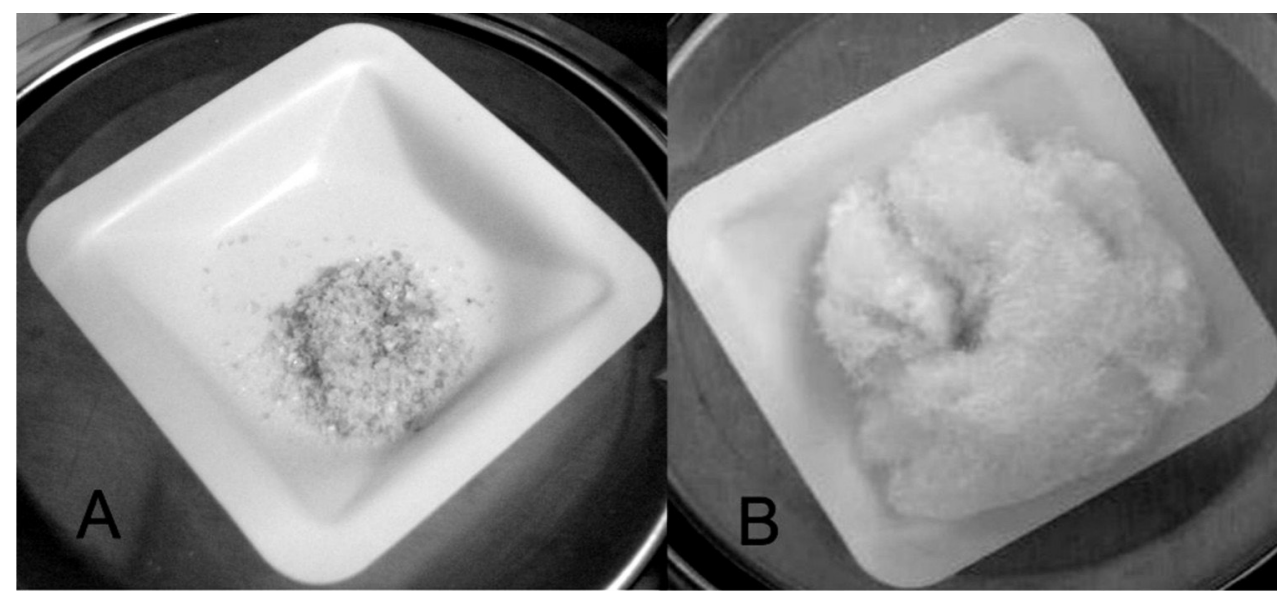

Figura 1S. (A) Ilustração do EPS $R_{1}$ seco e moído; (B) EPS $R_{1}$ dialisado e liofilizado 
A)

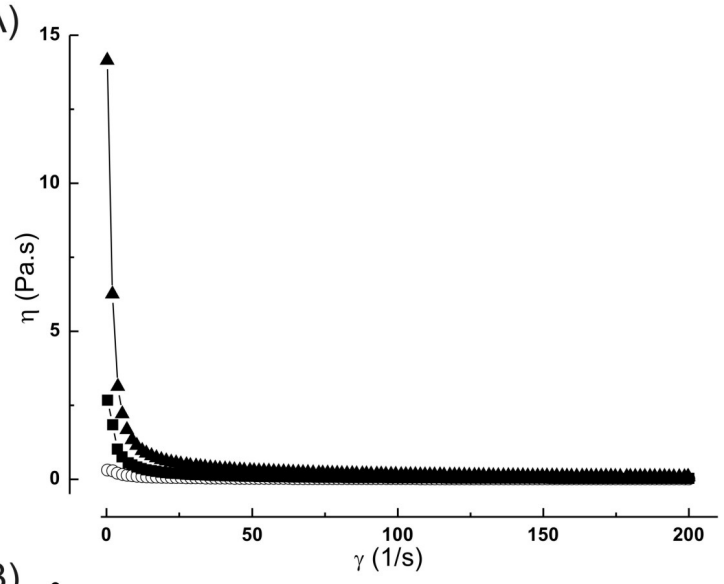

B)

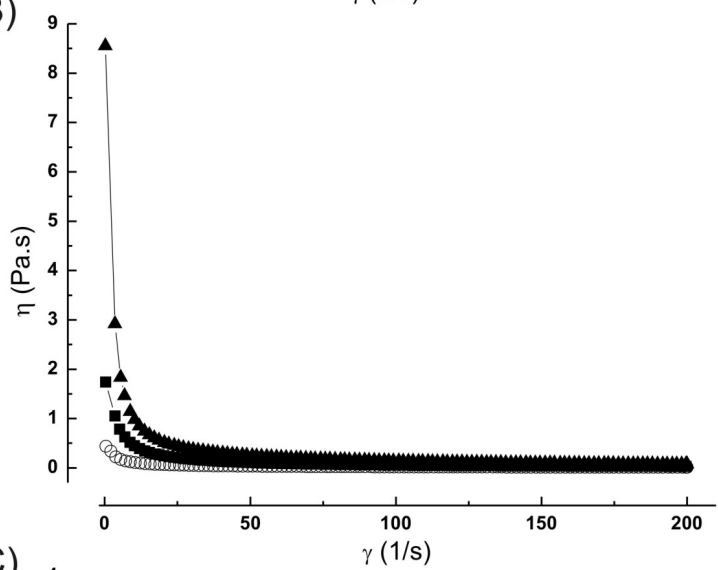

C)

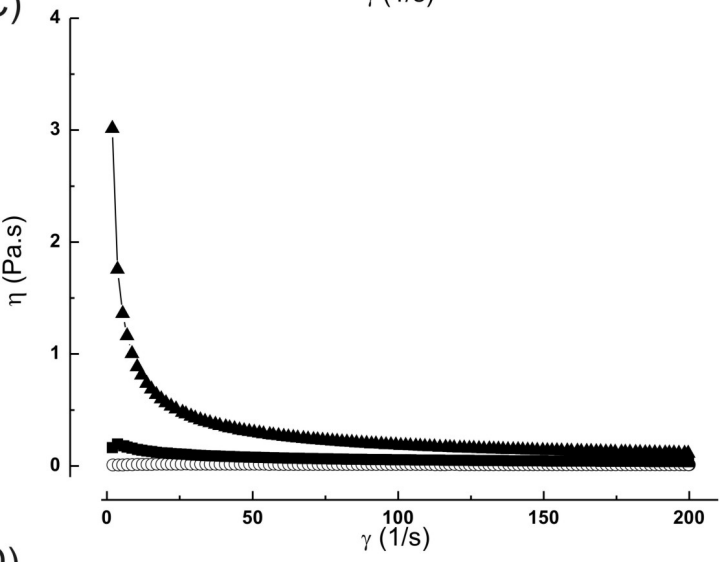

D)

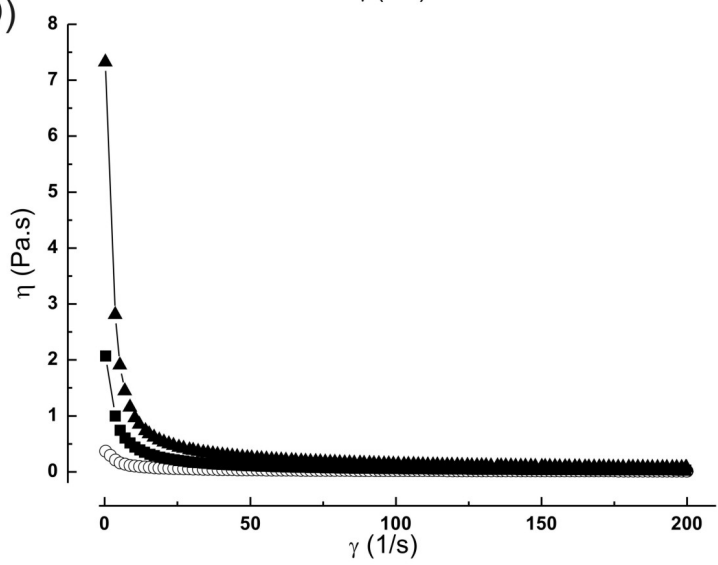

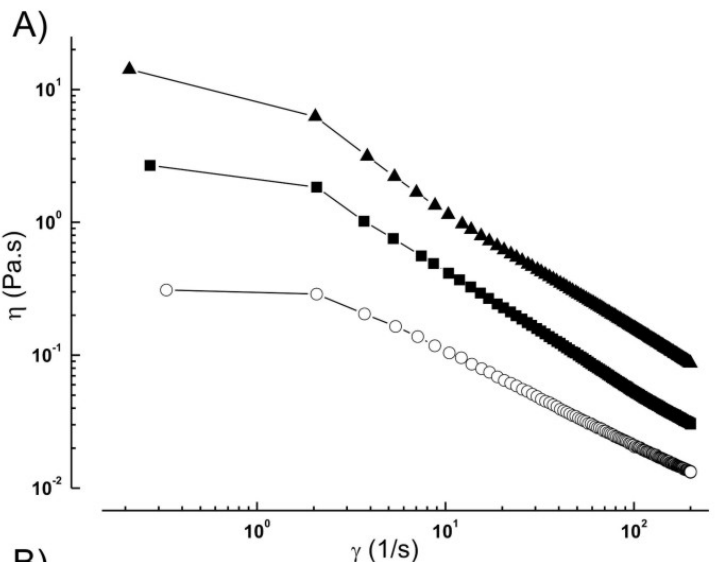

B)
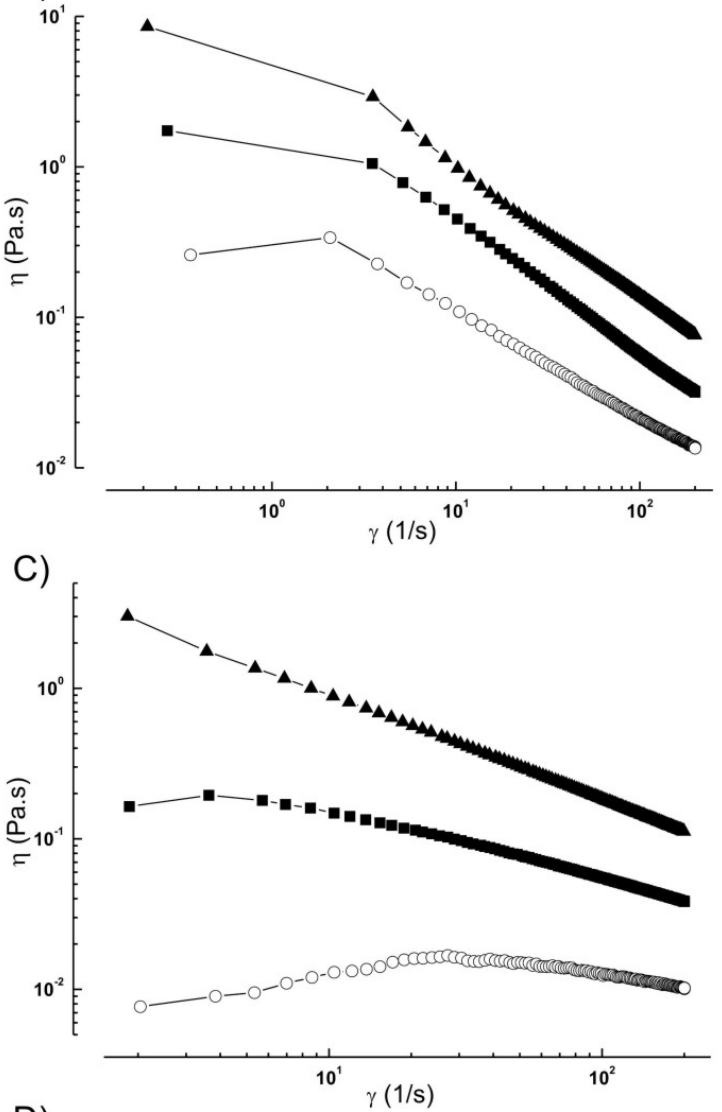

D)

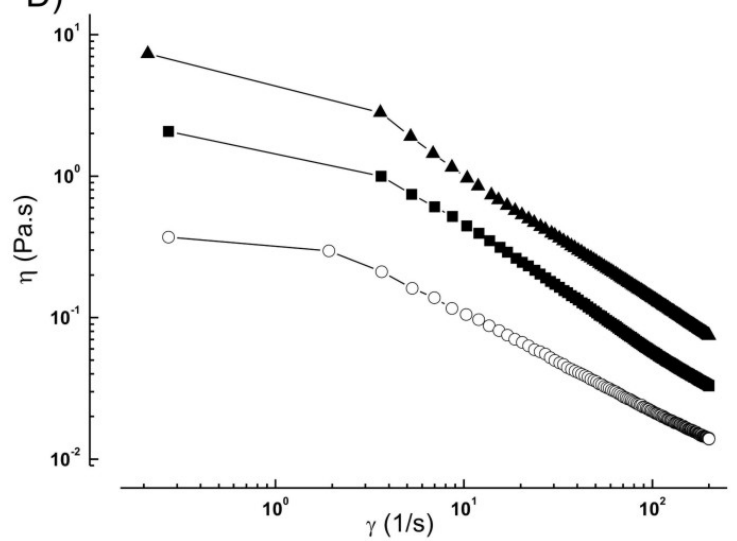

Figura 2S. Viscosidade aparente $(\eta)$ em função da taxa de deformação $(\dot{\gamma})$ de (A) $R_{l},(B) R_{2}$, (C) $R_{3}$ e (D) $R_{4}$ nas concentrações de $2 \mathrm{~g} / \mathrm{L}$ (-O-), $5 \mathrm{~g} / \mathrm{L}$ (--) e $10 \mathrm{~g} / \mathrm{L}(-\mathbf{-}-)$, em solução aquosa, à temperatura de $25^{\circ} \mathrm{C}$

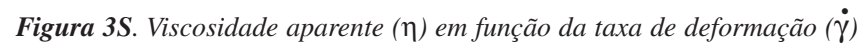
de (A) $R_{l}$, (B) $R_{2}$, (C) $R_{3}$ e (D) $R_{4}$ nas concentrações de $2 \mathrm{~g} / \mathrm{L}$ (-O-), $5 \mathrm{~g} / \mathrm{L}$ (-匹-) e $10 \mathrm{~g} / \mathrm{L}\left(-\mathbf{\Delta -}\right.$ ), em solução aquosa, à temperatura de $25^{\circ} \mathrm{C}$ 